\title{
Multi-objective Optimization Algorithms with the Island Metaheuristic for Effective Project Management Problem Solving
}

\author{
Christina BRESTER, Ivan RYZHIKOV, Eugene SEMENKIN
}

\begin{abstract}
Reshetnev Siberian State University of Science and Technology, Institute of Computer Science and Telecommunications, 31 »Krasnoyarskiy Rabochiy« ave., Krasnoyarsk, 660037, Russian Federation eugenesemenkin@yandex.ru (corresponding author)
\end{abstract}

\begin{abstract}
Background and Purpose: In every organization, project management raises many different decision-making problems, a large proportion of which can be efficiently solved using specific decision-making support systems. Yet such kinds of problems are always a challenge since there is no time-efficient or computationally efficient algorithm to solve them as a result of their complexity. In this study, we consider the problem of optimal financial investment. In our solution, we take into account the following organizational resource and project characteristics: profits, costs and risks.

Design/Methodology/Approach: The decision-making problem is reduced to a multi-criteria 0-1 knapsack problem. This implies that we need to find a non-dominated set of alternative solutions, which are a trade-off between maximizing incomes and minimizing risks. At the same time, alternatives must satisfy constraints. This leads to a constrained two-criterion optimization problem in the Boolean space. To cope with the peculiarities and high complexity of the problem, evolution-based algorithms with an island meta-heuristic are applied as an alternative to conventional techniques.

Results: The problem in hand was reduced to a two-criterion unconstrained extreme problem and solved with different evolution-based multi-objective optimization heuristics. Next, we applied a proposed meta-heuristic combining the particular algorithms and causing their interaction in a cooperative and collaborative way. The obtained results showed that the island heuristic outperformed the original ones based on the values of a specific metric, thus showing the representativeness of Pareto front approximations. Having more representative approximations, decision-makers have more alternative project portfolios corresponding to different risk and profit estimations. Since these criteria are conflicting, when choosing an alternative with an estimated high profit, decision-makers follow a strategy with an estimated high risk and vice versa.

Conclusion: In the present paper, the project portfolio decision-making problem was reduced to a 0-1 knapsack constrained multi-objective optimization problem. The algorithm investigation confirms that the use of the island meta-heuristic significantly improves the performance of genetic algorithms, thereby providing an efficient tool for Financial Responsibility Centres Management.
\end{abstract}

Keywords: 0-1 multi-objective constrained knapsack problem; project management portfolio problem; multi-objective evolution-based optimization algorithms; collaborative and cooperative meta-heuristics 


\section{Introduction}

When managing an organization, many different kinds of problems can be faced and a large proportion of these can be solved mathematically. These problems are actually decision-making problems in the space of alternatives and thus can be reduced to mathematical programming problems in which a solution that provides an extremum value of some criterion is a decision. The aim of decision-making support systems is to solve these mathematical programming problems so that managers could base their decisions on numerical analysis performed by the program software. This means that a computational system which supports the decision-making process for top managers in the project management problem is important, useful and its application provides a mathematically determined solution. In this paper, we focus on the problem, which takes place in machine-building factory management, where the project investment problem should be solved. According to this, we need to allocate funds among different financial responsibility centres.

In this study, we consider the two-objective knapsack problem, which is in some way similar to a real investment portfolio management problem for a factory. Here a factory, considered as a system, contains different subsystems with their specific products, functionality and properties. In big companies, there are many innovative projects aimed at modernizing technology, thus increasing income, reducing the amount of work in progress and making the business more client-oriented. Therefore, by solving this problem, it becomes possible to reduce the time spent by top managers on making decisions - their projects should be accepted and realized in the near future. It is important and should be highlighted that the characteristics of each subsystem and the complexity of project domains prevent people from being experts in all areas and, consequently, from making a properly weighted and informed decision. This explains the importance and the value of decision-making support systems with a growing focus on algorithms solving related problems.

The problem discussed in this paper differs from the ones in Markowitz's (Markovitz, 1952) modern portfolio theory based on mean-variance analysis, and also from those discussed in post-modern portfolio theory (Rom and Ferguson, 1993). It has the form of the decision-making multi-objective optimization problem, specifically, the two-criterion 0-1 knapsack optimization problem with constraints. The growing complexity, which is caused by growth in the problem dimensionality, nonlinearities, the specific nature of alternatives' representations and the multimodality of criteria, requires new algorithms which allow these difficulties to be overcome. Such algorithms are so-called evolution-based and nature-inspired techniques - stochastic optimization algorithms modified by many researchers to deal with complex problems. These mod- ifications change the algorithm operators, the algorithm structure or the meta-heuristics controlling the behaviour of the extremum-seeking algorithm.

There are many different approaches proposed for solving those portfolio problems in which various modifications are implemented. One of them is based on genetic algorithms (Goldberg, 1989) and an entropy-based modification (Aslan et al., 2015) which finds a solution in mean-variance terms. In the study (Drezewski and Doroz, 2017), the multi-agent co-evolutionary approach is applied to a portfolio multi-criteria optimization problem, and the genetic algorithm here is the main optimization technique. A combination of a genetic algorithm and particle swarm optimization (Kennedy and Eberhart, 1995) for solving this kind of problems is considered in (Kuo and Hong, 2013). The results of these investigations show that meta-heuristics greatly improve the performance of the algorithm.

Multi-objective knapsack optimization problems are still of vital importance. Many different approaches are applied, combined and developed for solving these problems which arise from decision-making problems of different backgrounds in various organizations. In the paper (Vianna and Vianna, 2013) a specific optimization algorithm based on a greedy-randomized adaptive search procedure (Feo and Resende, 1995) and a multi-objective iterated local search is proposed. In this work, many different multi-objective optimization methods were presented and one of them was the Chebyshev-based modification of a genetic algorithm (Alves and Almeida, 2007). In the study (Florios et al., 2010) some different approaches based on genetic algorithms were investigated for solving the considered problem.

In this study, we compare some cooperative approaches with homogenous and heterogeneous combinations joined in the island model for solving the project management decision-making problem for a machine-building factory. The experimental results prove that the proposed meta-heuristics outperform standard multi-criteria optimization algorithms.

\section{Project Portfolio and 0-1 Knapsack Multi-Criteria Problem}

One of the common ways of alternative space representation in the 0-1 knapsack problem, related to project portfolio management, is the Boolean space $B^{n}$, where $n$ is a space dimension and is equal to the number of projects. In other words, the way the knapsack is packed (portfolio of projects), is a Boolean vector $x \epsilon B^{n}$ in which coordinates are decisions on each project: it is 0 or false if we decline the project realization and it is 1 or true if we accept the project. In this study, we consider an organization which structurally consists of $m$ financial responsibility centres 
(FRC) and there are

$$
N_{j}, j=\overline{1, m}
$$

different projects for each FRC. The whole number of projects

$$
n=\sum_{j=1}^{m} N_{j}
$$

determines the dimensionality of the Boolean space.

In this decision-making problem, the $j$-th project of $i$-th FRC has the following characteristics: $c_{i, j}$ denotes the annual costs of the project realization, $R_{i, j}$ is an expert's estimation of its realization risks and $P_{i, j}$ is the annual profit of the project if it is accepted. The whole organization also has its own characteristics: $C$ is the total amount of credits, which is normally a sum of $C_{i}$, the annual credits of each FRC for project realizations and $\hat{C}$, which is the same for the whole organization. We may also require the specific rate of return on capital $r$ to be satisfied.

This problem definition leads to a pseudo-Boolean optimization problem with two criteria and inequality constraints. The first criterion is the maximization of the profit of all the accepted projects and the second criterion is the minimization of the sum of the risks. As can be seen, the first criterion is to be maximized, and the second - minimized:

$$
\begin{aligned}
& F_{1}(x)=\sum_{i=1}^{m} \sum_{j=1}^{N_{i}} P_{i, j} \cdot x_{I(i, j)} \rightarrow \max _{x \in B^{n}}, \ldots: \\
& F_{2}(x)=\sum_{i=1}^{m} \sum_{j=1}^{N_{i}} R_{i, j} \cdot x_{I(i, j)} \rightarrow \min _{x \in B^{n}}, \cdots:
\end{aligned}
$$

where

$$
I(i, j)=\sum_{k=0}^{i-1} N_{k}+j, N_{0}=0
$$

is a specific indexing function which returns the index of a Boolean vector for the $j$-th project of the $i$-th FRC.

At the same time, the project portfolio must satisfy the constraints. We cannot exceed the amount of credits and we cannot go below the current return rate on capital:

$$
\begin{aligned}
& B_{1}(x)=\sum^{m} \sum^{N_{i}} c_{i, j} \cdot x_{I(i, j)} \leq C, C=\sum^{m} C_{i}+\hat{C}, \\
& B_{2}(x)=F_{1}(x) / B_{1}(x) \geq r .
\end{aligned}
$$

To reduce the constrained extremum-seeking problem (1)-(4) to an unconstrained one, we use the static penalty functions:

$$
F_{p}(z)= \begin{cases}z & \text { if } z>0 \\ 0 & \text { if } z<0\end{cases}
$$

and the initial problem can be determined with the formulas:

$$
\begin{aligned}
& \hat{F}_{1}(x)=F_{1}(x)-\alpha_{1,1} \cdot F_{p}\left(B_{1}(x)-C\right)-\cdots \\
& \quad \cdots \alpha_{1,2} \cdot F_{p}\left(B_{2}(x)-r\right) \rightarrow \max _{x \in B^{n}}, \cdots \\
& \hat{F}_{2}(x)=F_{2}(x)+\alpha_{2,1} \cdot F_{p}\left(B_{1}(x)-C\right)+\cdots \\
& \quad \cdots \alpha_{2,2} \cdot F_{p}\left(B_{2}(x)-r\right) \rightarrow \min _{x \in B^{n}}, \ldots \ldots
\end{aligned}
$$

where positive numbers

$$
\alpha_{i, j}, i, j=\overline{1,2}
$$

are the controlling parameters. In this study, we set all the parameters equal to $\alpha=10^{3}$.

The considered problem (6) and (7) is known to be NP-hard so there is no time and computational-efficient optimization technique that would find a global optimum. This becomes further complicated when we need to find the set of solutions which approximates the Pareto set. As was mentioned earlier, we need a specific optimization technique which is efficient in solving this kind of problem, and for this purpose we use modern multi-objective algorithms and improve them with the island meta-heuristic (Preuss, 2015).

\section{Multi-Objective Genetic Algorithms and the Island Model Meta- Heuristic}

The common scheme of any multi-objective genetic algorithm (MOGA) includes the same steps as any conventional one-criterion GA (Crainic and Toulouse, 2010):

1 Generate the initial population

2 Evaluate criteria values;

3 Estimate fitness-values;

4 While (stop-criterion!=true), do:

\{

5 Choose the most appropriate individuals with the mating selection operator based on their fitnessvalues:

6 Produce new candidate solutions with recombination;

7 Modify the obtained individuals with mutation;

8 Evaluate criteria values for new candidate solutions;

9 Estimate fitness-values;

10 Compose the new population (environmental selection);

\} 
When designing a MOGA, researchers are faced with some issues relating to fitness assignment strategies, diversity preservation techniques and ways of elitism implementation. Therefore, we will consider the effectiveness of MOGAs which are based on various heuristics. Non-Sorting Genetic Algorithm II (NSGA-II) (Deb et al., 2002), Preference-Inspired Co-Evolutionary Algorithm with goal vectors (PICEA-g) (Wang, 2013) and Strength Pareto Evolutionary Algorithm 2 (SPEA2) (Zitzler et al., 1997) are used as tools to optimize the introduced criteria. The basic features of each method are displayed in Table 1.
However, it is almost impossible to know in advance which algorithm is the most effective for the current problem. On the one hand, a series of experiments might be conducted to find the best MOGA, which is quite a time-consuming approach. On the other hand, different algorithms might be combined in a cooperation to avoid having to choose the most effective one. In reality, this kind of modification is easily implemented and is based on an island model.

The island model (Whitley et al., 1997) of a GA implies the parallel work of several algorithms: they might

Table 1: Basic features of the MOGA used

\begin{tabular}{|c|c|c|c|}
\hline MOGA & Fitness Assignment & Diversity Preservation & Elitism \\
\hline NSGA-II & $\begin{array}{c}\text { Pareto-dominance (niching mecha- } \\
\text { nism) and diversity estimation (crowd- } \\
\text { ing distance) }\end{array}$ & Crowding distance & $\begin{array}{c}\text { Combination of the previous } \\
\text { population and the offspring }\end{array}$ \\
\hline PICEA-g & $\begin{array}{c}\text { Pareto-dominance (with generating } \\
\text { goal vectors) }\end{array}$ & $\begin{array}{c}\text { Nearest neighbour } \\
\text { technique }\end{array}$ & $\begin{array}{c}\text { The archive set and combina- } \\
\text { tion of the previous popula- } \\
\text { tion and the offspring }\end{array}$ \\
\hline SPEA2 & $\begin{array}{c}\text { Pareto-dominance (niching mech- } \\
\text { anism) and density estimation }(\text { the } \\
\text { distance to the } \text { k-th nearest neighbour } \\
\text { in the objective space) }\end{array}$ & $\begin{array}{c}\text { Nearest neighbour } \\
\text { technique }\end{array}$ & The archive set \\
\hline
\end{tabular}

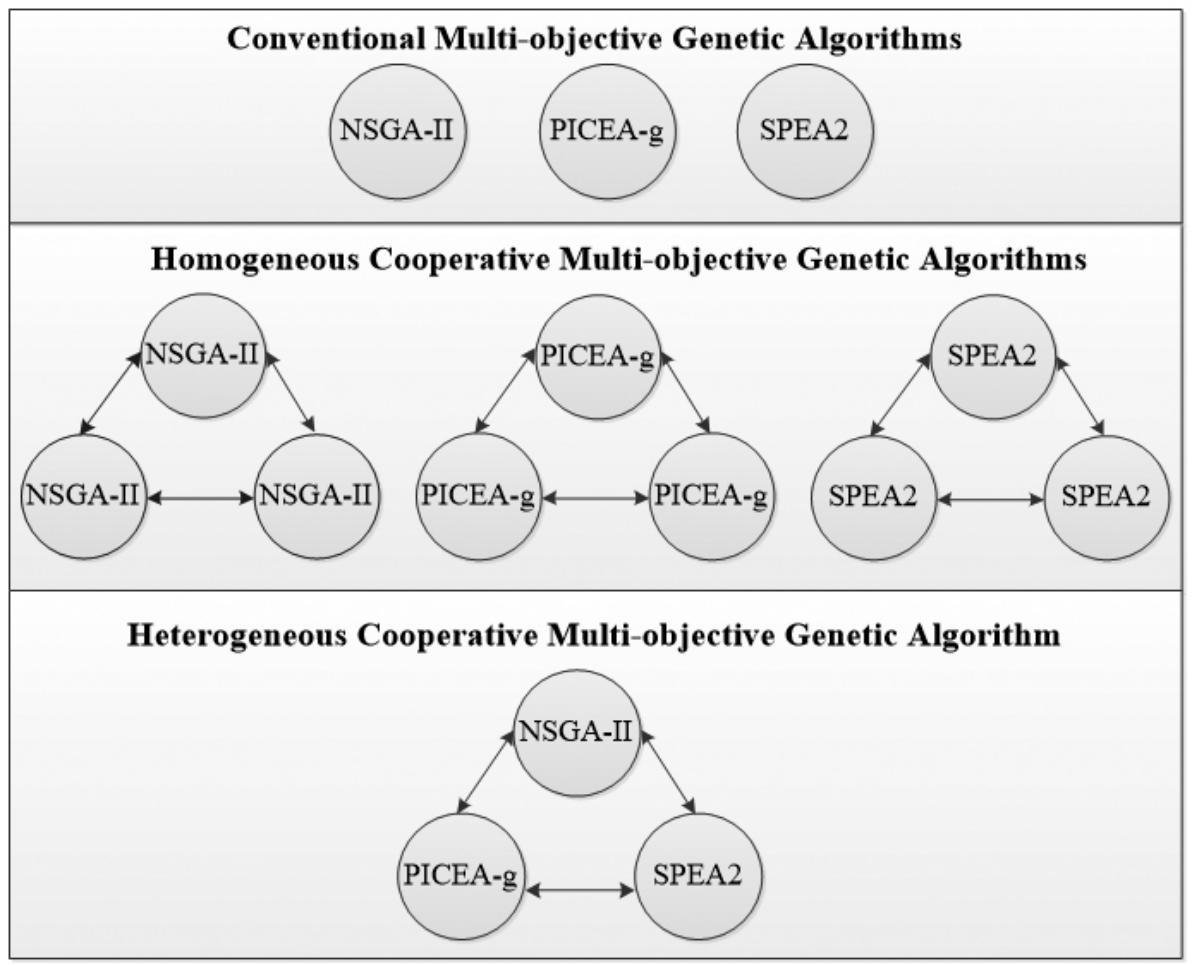

Figure 1: The three categories of algorithms used 
be the same or different. The initial number of individuals $M$ is spread across $L$ subpopulations: $M_{i}=M / L, i=1, \ldots$, $L$. At each $T$-th generation, algorithms exchange the best solutions (migration). There are two parameters: migration size, the number of candidates for migration, and migration interval, the number of generations between migrations. It is also necessary to define the island model topology, in other words, the scheme of migration. We use fully connected topology, meaning that each island shares its best solutions with all the other islands included in the model. This multi-agent model is expected to preserve a higher level of genetic diversity.

Firstly, the conventional NSGA-II, PICEA-g, and SPEA2 have been implemented to be used as optimizers (Figure 1 top).

Secondly (Figure 1, middle), we have achieved a number of homogeneous cooperative algorithms: in each case, the island model has the same three components: they are NSGA-II, PICEA-g or SPEA2. In addition to diversity preservation, another benefit of this model is the possibility to reduce the computational time due to the parallel work of islands.

Finally, a heterogeneous cooperative algorithm has been developed (Figure 1, bottom). Three different MOGAs (NSGA-II, PICEA-g and SPEA2) have been included in this model as its components simultaneously. The benefits of the particular algorithm (NSGA-II, PICEA-g or SPEA2) could be advantageous at different stages of optimization (Brester and Semenkin, 2015).

In summary, there are three main categories of MOGAs which are used in this study and they are portrayed in Figure 1.

\section{Statistical Investigations}

The problem in question was solved for a big machine-building plant. There were five FRC $(m=5)$ and each FRC had its own list of projects and required investments $\left(N_{1}=8, N_{2}=6, N_{3}=5, N_{4}=3, N_{5}=3\right)$.

$$
\sum_{j=1}^{m} N_{j}=25
$$

For each of the projects, we had an expert's estimations of the risks $R_{i, j}$ and annual profits $P_{i, j}$. The whole number of projects $n$ was equal to

hence in this knapsack problem we had 25 Boolean variables. Other parameters were set as follows: $\hat{C}=40, \mathrm{r}=0.5$.

Firstly, we used an exhaustive search to design a true Pareto front. It required $2^{25}=33554432$ vector-function evaluations. In Figure 2, the obtained front is presented. It might be noted that the dependence between the $F_{1}$ - (6) and $F_{2}$ - (7) criteria is close to linear. Increasing the profit would cause an increasing in the risk, and minimizing the risk leads to a decrease in profit.

It is essential to note that for an exhaustive search, an increase in the problem dimensionality leads to the exponential growth of vector-function evaluations. Therefore, for high-dimensional problems, it might be time-consuming and some alternative methods should be developed.

Next, we applied the conventional NSGA-II, PICEA-g and SPEA2 to solve the problem. In all the experiments, we defined the following settings: binary tournament selection, uniform recombination and the mutation probability $p_{m}=1 / L$, where $L$ is the length of the chromosome. A series of tests with different amounts of resources was conducted: Exp. 1 - 100 individuals and 200 generations (20,000 vector-function evaluations), Exp. 2 - 200 individuals and 300 generations (60,000 vector-function evaluations), Exp. 3 - 300 individuals and 400 generations $(120,000$ vector-function evaluations). To estimate the quality of the obtained approximations of the true front, we

$$
\operatorname{IGD}\left(P^{*}, A\right)=\frac{\sum_{v \in P^{*}} d(v, A)}{\left|P^{*}\right|}
$$

involved Inverted Generational Distance (IGD) (8), which equates the average distance from the true Pareto front $P^{*}$ to the found solution $A$ (Zhang et al., 2008):

where $d(v, A)$ is the minimum Euclidean distance between $v$ and the points in $A$.

All the results were averaged over 25 runs of each algorithm. Table 2 contains the averaged IGD values corresponding to three experiments (Exp. 1,2 and 3) and three conventional MOGAs (NSGA-II, PICEA-g and SPEA2).

By increasing the amount of resources, we obtain approximations which are getting closer to the true front. In two cases (for PICEA-g and SPEA2), we may see a great improvement of IGD values caused by the growth of vector-function evaluations. For NSGA-II, increasing the amount of resources by a factor of two does not lead to a significant improvement (from 20,000 up to 60,000 ) or to any improvement (from 60,000 up to 120,000 ). The algorithm which was the best for the lowest number of vector-function evaluations (Exp. 1) was the worst for the highest number of calculations (Exp. 3).

To illustrate the obtained solutions, from each experiment we chose one Pareto front approximation corresponding to the median value of IGD. In Figure 3, we depict these approximations.

Then, we applied three homogeneous cooperative MOGAs: NSGA-II - NSGA-II - NSGA-II, PICEA-g PICEA-g - PICEA-g and SPEA2 - SPEA2 - SPEA2. For each MOGA, all islands had an equal amount of resources (200 generations and $300 / 3=100$ individuals in populations), the migration size was equal to 20 (in total, each island received 40 points from two others), and the migration interval was equal to 20 generations. Thus, in this experiment the amount of resources corresponded to the 


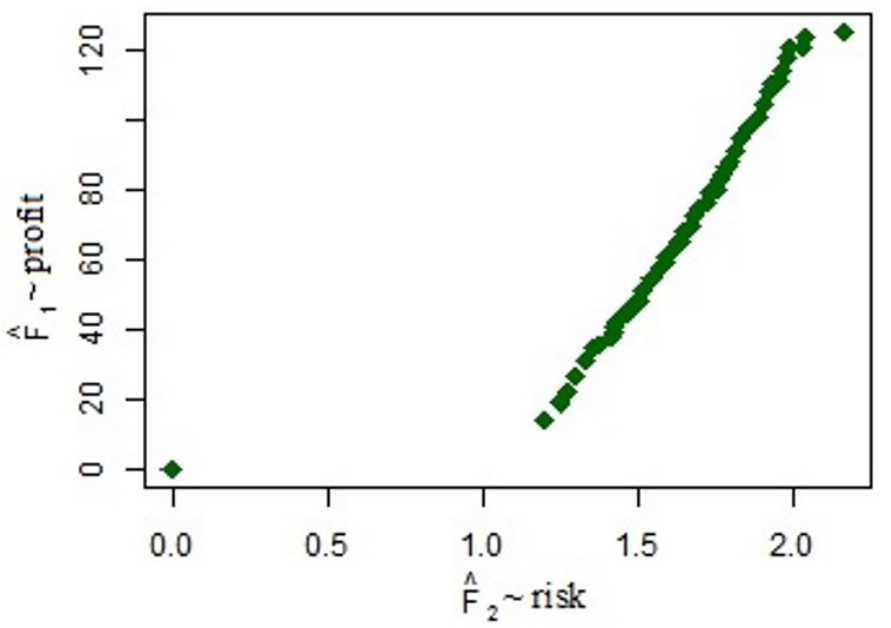

Figure 2: The true Pareto front for the real problem considered

Table 2: Experimental results. IGD values for the conventional MOGAs

\begin{tabular}{|c|c|c|c|}
\hline \multirow{2}{*}{ MOGA } & \multicolumn{3}{|c|}{ IGD values } \\
\cline { 2 - 4 } & Exp. 1 (20,000 eval.) & Exp. 2 (60,000 eval.) & Exp. 3 (120,000 eval.) \\
\hline NSGA-II & 0.5520 & 0.4664 & 0.4838 \\
\hline PICEA-g & 0.9649 & 0.5598 & 0.3564 \\
\hline SPEA2 & 0.7352 & 0.4423 & 0.2822 \\
\hline
\end{tabular}
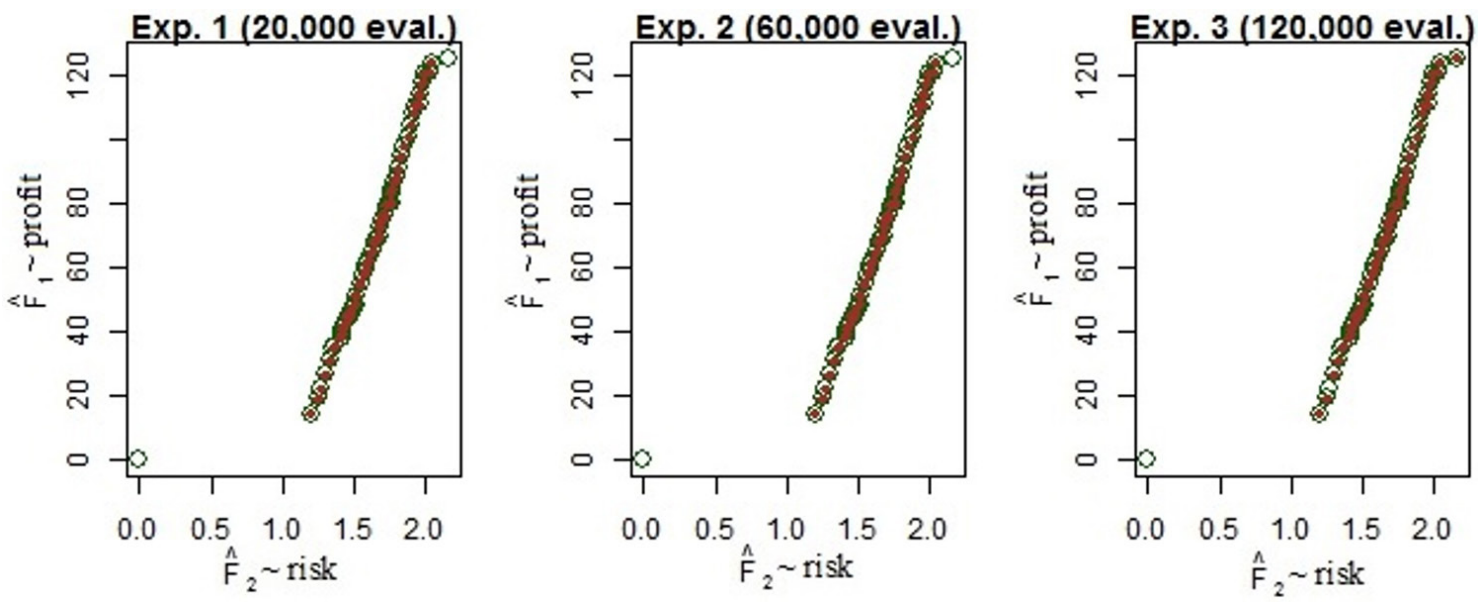

Figure 3a: The Pareto front approximations obtained by NSGA2

number of vector-function evaluations in Exp. 2 (60,000). We also estimated the averaged IGD values and presented them in Table 3. It might be noted that the use of the island model led to a considerable improvement in IGD values. Moreover, having the same amount of resources as we had in Exp. 2, we could achieve IGD values which were comparable with (for PICEA-g and SPEA2) or even better (for NSGA-II) than we gained in Exp. 3.
Finally, the heterogeneous MOGA (NSGA-II - PICEA-g - SPEA2) was used to solve the problem in question. Again, we provided the algorithm with 60,000 vector-function evaluations. All the other settings were also the same (as for homogeneous cooperative MOGAs). The averaged IGD value obtained by the heterogeneous cooperative MOGA is equal to the best averaged IGD value achieved by the homogeneous cooperative MOGA (Table 

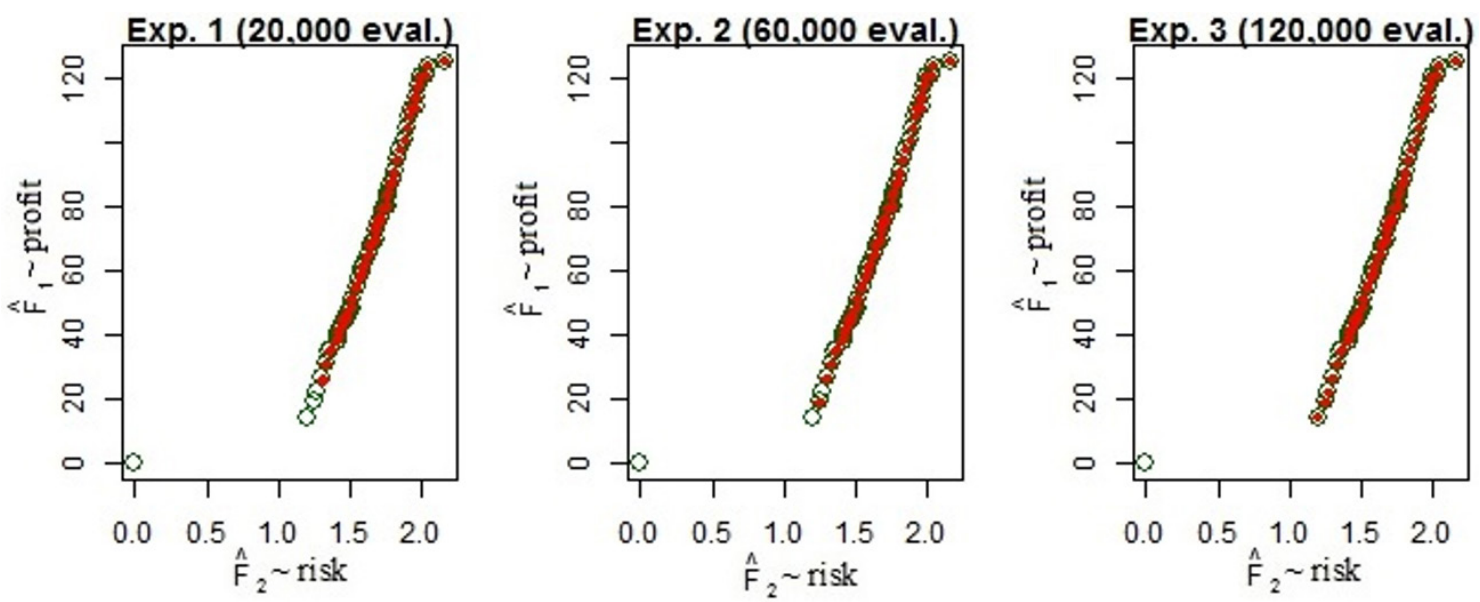

Figure 3b: The Pareto front approximations obtained by PICEA-g
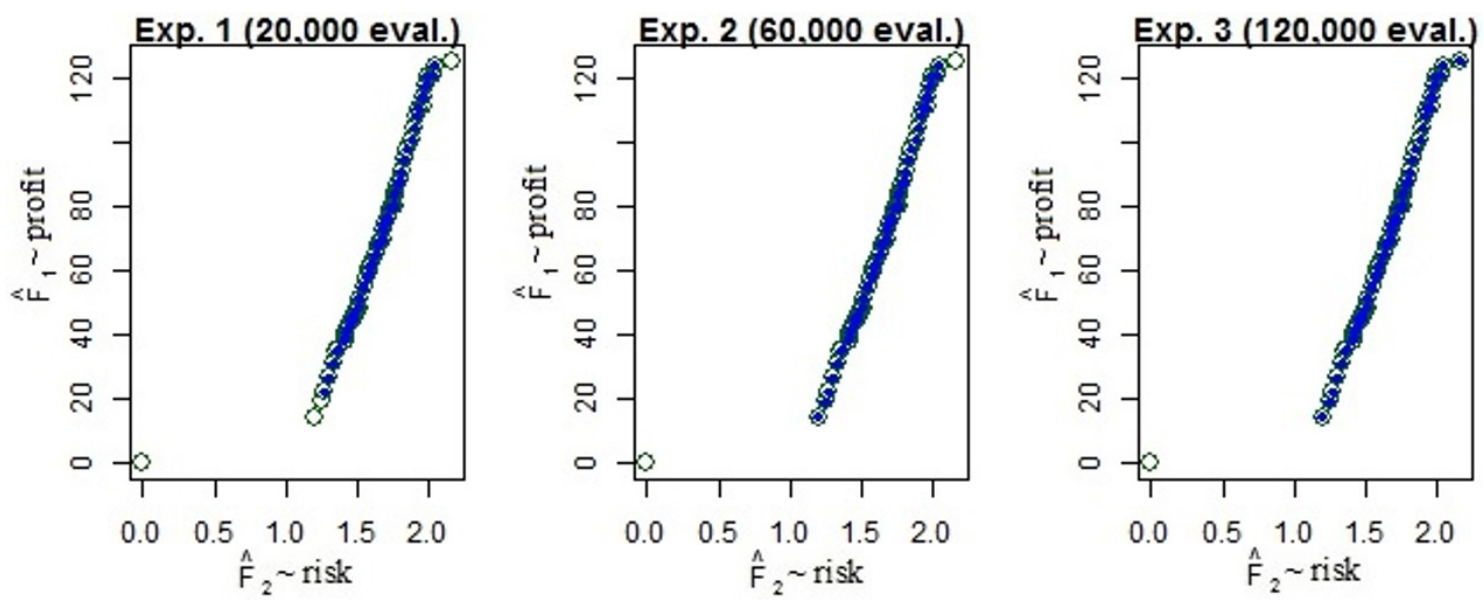

Figure 3c: The Pareto front approximations obtained by SPEA2

Table 3: Experimental results. IGD values for the cooperative MOGAs

\begin{tabular}{|c|c|}
\hline \multicolumn{2}{|c|}{ IGD values } \\
\hline \multicolumn{2}{|c|}{ Homogeneous cooperative MOGAs } \\
\hline NSGA-II - NSGA-II - NSGA-II & 0.2985 \\
\hline PICEA-g - PICEA-g - PICEA-g & 0.4153 \\
\hline SPEA2 - SPEA2 - SPEA2 & 0.3876 \\
\hline \multicolumn{2}{|c|}{ Heterogeneous cooperative MOGA } \\
\hline NSGA-II - PICEA-g - SPEA2 & $\mathbf{0 . 2 9 8 4}$ \\
\hline
\end{tabular}




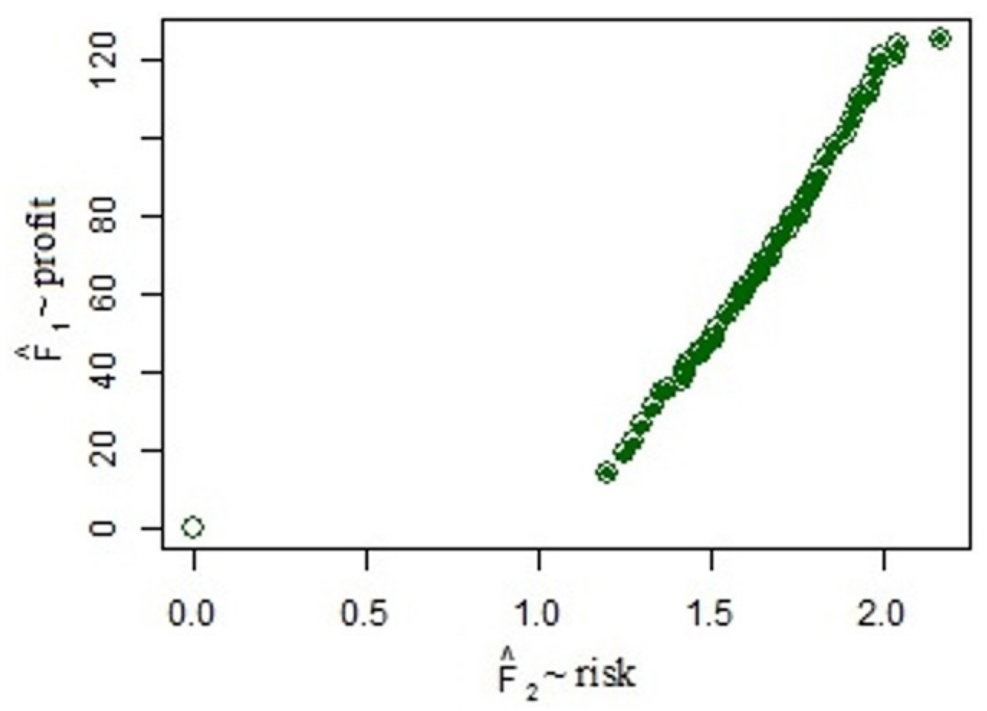

Figure 4. The Pareto front approximation obtained by the heterogeneous MOGA

3). It is also comparable with the best result in Exp. 3 (with 120,000 vector-function evaluations).

In Figure 4, we show one Pareto front approximation found by the heterogeneous cooperative MOGA and corresponding to the median value of IGD.

The results obtained proved the effectiveness of cooperative MOGAs: firstly, with the same amount of resources we could attain much better IGD values and, secondly, using the heterogeneous cooperative MOGA, we could avoid having to choose the most appropriate MOGA for the current problem (it is essential because MOGAs demonstrate different performances in Exp. 1, 2 and 3).

As one can see, the estimated Pareto front provides decision-makers with possible outcomes, in case they consider multiple criteria, and enables them to choose the combination, which would fit the current state of the market. The proposed heterogeneous island approach also provides faster convergence toward the solutions.

\section{Conclusion}

In this study, we focused on the decision-making problem related to machine-building factory portfolio management with the goal of optimal investment, which can be defined as the $0-1$ multi-objective constrained knapsack optimization problem. This problem is NP-hard, the criteria are mappings from the Boolean space and we need to estimate the Pareto front on a set of permissible alternatives. To reach the goal, an efficient multi-objective optimization technique is required.

We applied well-known evolution-based algorithms such as PICEA-g, SPEA2 and NSGA-2 for this problem with different amounts of resources. The algorithms were compared using the specific IGD metric, which is a common measure of Pareto front representativeness. As can be seen, increasing the computational resources usually yielded an increase in the efficiency of the algorithm and, with the exception of NSGA2, the increase is significant. Hence, adding more resources may improve the results, though the effect is unpredictable and non-linear. Moreover, in the case of NSGA2 being applied to this problem, the median of the IGD metric was not improved after 60,000 evaluations and this is probably a result of the algorithm behaviour.

To overcome this obstacle, we used an island model based on the interaction among multi-objective optimization algorithms: homogeneous, when the algorithms are of the same nature, and heterogeneous, when the algorithms are different. Experimental results show that the developed approach outperforms the original algorithms even with the lower amount of computational resources. The most efficient algorithms are the following: the heterogeneous algorithm with the SPEA2, NSGA-2 and PICEA-g combination and the homogeneous algorithm with three NSGA2. This implies that the island model-based multi-objective algorithms are more efficient and more promising in solving the complex NP-hard problems of organizational management.

The proposed approach provides us with a set of non-dominated alternatives, which are project portfolios with different profits and risks. This solution is valuable for top managers when they make decisions on future investments based on the current state of the whole organization and estimations of project characteristics. More profitable project portfolios usually have a high level of risks and less profitable project portfolios correspond to a low level of risks. The main benefit of applying the pro- 
posed approach is its flexibility and ability to show the bigger picture. Whatever risk value is confirmed by the decision-maker, the Pareto set approximation gives the best portfolio in terms of the profit and vice versa.

Our proposal is going to be investigated on higher-dimensional similar problems with nonlinear profit functions, since most of the projects are related and affect each other. This is the first possible direction of our research in the near future. Following this, it would be reasonable to solve similar problems with stochastic uncertainties as is considered in modern portfolio theory where risks and profits are the stochastic variables.

\section{Acknowledgment}

This research is performed with the financial support of the Ministry of Education and Science of the Russian Federation within state assignment № 2.6757.2017/БЧ.

\section{References}

Alves, M.J., \& Almeida, M. (2007). MOTGA: A multiobjective Tchebycheff based genetic algorithm for the multidimensional knapsack problem. Computers \& Operations Research, 34(11), 3458-3470, http://dx. doi.org/10.1016/j.cor.2006.02.008

Aslan, O., Mert Kantar, Y., \& Usta, I. (2015). Genetic algorithms for solving portfolio allocation models based on relative-entropy, mean and variance. Journal of Scientific Research and Development, 2 (12), 7-12. Retrieved from http://jsrad.org/wp-content/2015/ Issue $\% 2012, \% 202015 / 2 \mathrm{jj} . p d f$

Brester Ch. \& Semenkin E. (2015). Cooperative Multi-objective Genetic Algorithm with Parallel Implementation. Advances in Swarm and Computational Intelligence, LNCS vol. 9140. pp. 471-478. Springer Nature.

Crainic, T. G., Toulouse, M. (2010). Parallel metaheuristics. In Handbook of metaheuristics, pp. 497-541. Springer. Retrieved from https://link.springer.com/ chapter/10.1007/978-1-4419-1665-5_17

Deb, K., Pratap, A., Agarwal, S. \& Meyarivan, T. (2002). A fast and elitist multiobjective genetic algorithm: NSGA-II. IEEE Transactions on Evolutionary Computation, 6 (2), 182-197, https://doi. org/10.1109/4235.996017

Drezewski, R., \& Doro, K. (2017). An Agent-Based Co-Evolutionary Multi-Objective Algorithm for Portfolio Optimization, Symmetry, 9, 168, http://dx.doi. org/10.3390/sym9090168

Feo, T. A., \& Resende, M. G. C. (1995). Greedy randomized adaptive search procedures. Journal of Global Optimization, 6, 109-133, http://dx.doi.org/10.1007/ BF01096763

Florios, K., Mavrotas, G., \& Diakoulaki, D. (2010). Solv- ing multiobjective, multiconstraint knapsack problems using mathematical programming and evolutionary algorithms. European Journal of Operational Research, 203, (1), pp. 14-21, http://dx.doi.org/10.1016/j. ejor.2009.06.024

Goldberg, D. (1989). Genetic Algorithms in Search, Optimization and Machine Learning. Reading: MA: Addison-Wesley Professional.

Kennedy, J., \& Eberhart, R. (1995). Particle Swarm Optimization. Proceedings of IEEE International Conference on Neural Networks. IV. pp. 1942-1948, https:// doi.org/10.1109/ICNN.1995.488968

Kuo, R. J. \& Hong, C. W. (2013). Integration of Genetic Algorithm and Particle Swarm Optimization for Investment Portfolio Optimization. Appl. Math. Inf. Sci., 7(6), 2397-2408, http://dx.doi.org/10.12785/ amis/070633

Markowitz, H. (1952). Portfolio selection. J. Finance, 7, 77-91, http://dx.doi.org/10.1111/j.1540-6261.1952. tb01525.x

Rom, B. M., \& K. Ferguson. (1993). Post-Modern Portfolio Theory Comes of Age. Journal of Investing, Winter 1993. retrieved from http://www.actuaries.org/AFIR/ colloquia/Orlando/Ferguson_Rom.pdf

Preuss, M. (2015). Multimodal Optimization by Means of Evolutionary Algorithms, Springer International Publishing, http://dx.doi.org/10.1007/978-3-319-07407-8

Vianna, D. S., Vianna, M. F. D. (2013). Local search-based heuristics for the multiobjective multidimensional knapsack problem. Produção, 23(3), 478-487, http:// dx.doi.org/10.1590/S0103-65132012005000081

Wang, R. (2013). Preference-inspired co-evolutionary algorithms. A thesis submitted in partial fulfilment for the degree of the Doctor of Philosophy, University of Sheffield, p. 231.

Whitley, D., Rana, S. \& Heckendorn, R. (1997). Island model genetic algorithms and linearly separable problems. Proceedings of AISB Workshop on Evolutionary Computation, Manchester, UK. Springer, volume 1305 of LNCS, pp. 109-125.

Zhang, Q., Zhou, A., Zhao, S., Suganthan, P. N., Liu, W., Tiwari, S. (2008). Multi-objective optimization test instances for the CEC 2009 special session and competition. University of Essex and Nanyang Technological University, Tech. Rep. CES-487. Retrieved from http://dces.essex.ac.uk/staff/zhang/MOEAcompetition/cec09testproblem0904.pdf

Zitzler, E., Laumanns, M. \& Thiele, L. (2002). SPEA2: Improving the Strength Pareto Evolutionary Algorithm for Multiobjective Optimization. Evolutionary Methods for Design Optimisation and Control with Application to Industrial Problems EUROGEN 2001, 3242 (103), pp. 95-100. 
Christina Brester received her Master's degree in System Analysis and Control from the Reshetnev Siberian State Aerospace University (Krasnoyarsk, Russia) in 2014 and her PhD in Computer Science from the Siberian Federal University and the Institute of Computational Modeling of Siberian Branch of Russian Academy of Sciences (Krasnoyarsk, Russia) in 2016. Since 2016, she has been an Associate Professor at the Higher Mathematics Department of the Institute of Computer Science and Telecommunications of the Reshetnev Siberian State University of Science and Technology (Krasnoyarsk, Russia). Her areas of research include multi-objective optimization, neural networks and data analysis.

Ivan Ryzhikov received his Master's degree in System Analysis and Control from the Reshetnev Siberian State Aerospace University (SibSAU), Krasnoyarsk, Russia in 2011. He received his $\mathrm{PhD}$ in Computer Science from SibSAU in 2016. Since 2011, he has been a research fellow at the Reshetnev Siberian State University of Science and Technology in Krasnoyarsk, Russia. His research interests include metaheuristic optimization, inverse modelling, data science and computer science.

Eugene Semenkin received his Master in Applied Mathematics degree from Kemerovo State University (Kemerovo, USSR) in 1982, his PhD in Computer Science from Leningrad State University (Leningrad, USSR) in 1989 and his DSc in Engineering and Habilitation from the Siberian State Aerospace University (Krasnoyarsk, Russia) in 1997. Since 1997, he has been a professor of systems analysis at the Institute of Computer Science and Telecommunications of the Siberian State Aerospace University. His areas of research include the modelling and optimization of complex systems, computational intelligence and data mining. He has been awarded the Tsiolkovsky Badge of Honour by the Russian Federal Space Agency and the Reshetnev medal by the Russian Federation of Cosmonautics. 